\title{
Obesidad mórbida asociada a quiste broncogénico y leiomioma subcardial que simula GIST. Caso clínico*
}

\author{
Drs. JAIME RAPPOPORT S. ${ }^{1}$, GUILLERMO WATKINS S. ${ }^{1}$, ATTILA CSENDES J. ${ }^{1}$,
} MANUEL LILLO C. ${ }^{1}$, IVÁN GALLEGOS M. ${ }^{2}$, Int. DANIEL RAPPOPORT W. ${ }^{3}$

Departamento de Cirugía. Hospital Clínico, Universidad de Chile.

2 Servicio de Anatomía Patológica Hospital Clínico, Universidad de Chile.

3 Interno Facultad de Medicina, Universidad de Chile, Santiago, Chile.

\section{Abstract}

\section{Morbid obesity, esophageal bronchogenic cyst and subcardial leiomioma. Report of one case}

Gastric wall diseases are found in approximately of $1 \%$ of the patients submitted to bariatric surgery. Half of these are leiomiomas. Esophageal bronchogenic cysts are exceptional We report a 57 years old female with morbid obesity, a bronchogenic esophageal cyst and subcardial leiomioma. The preoperative study and intraoperative biopsy, suggested the presence of a gastrointestinal stromal tumor (GIST), but the definitive pathological study did not confirm its presence. A total gastrectomy was performed, with an uneventful postoperative course.

Key words: GIST, morbid obesity, bronchogenic esophageal cyst, leiomioma, total gastrectomy.

\section{Resumen}

La patología intramural gástrica tiene una frecuencia menor al 1\%, en cirugía gástrica. Alrededor del $50 \%$ corresponden a leiomiomas. Los quistes broncogénicos tienen origen embriológico y son excepcionales, y la mayor parte de los reportes corresponden a pacientes pediátricos. Se presenta un caso clínico de paciente portadora de Obesidad mórbida $(\mathrm{IMC}=52)$, asociado a lesión subcardial que simula GIST, cuyo estudio histopatológico resultó ser un quiste esofágico de origen broncogénico de $40 \mathrm{~mm}$, asociado a leiomioma subcardial de $10 \mathrm{~mm}$. Fue sometida a gastrectomía total más anastomosis esófago-yeyunal en Y de Roux, con asa de $180 \mathrm{~cm}$. No presentó morbi-mortalidad postoperatoria. Se discuten los métodos de estudio preoperatorio y las alternativas terapéuticas. No hemos encontrado en la literatura otro caso de asociación de estas raras patologías. total.

Palabras clave: GIST, obesidad mórbida, quiste broncogénico esofágico, leiomioma, gastrectomía

\footnotetext{
Correspondencia: Dr. Jaime Rappoport S.

Santos Dumont 999, Santiago, Chile

E-mail: rapaj@usa.net
}

*Recibido el 4 de Junio de 2008 y aceptado para publicación el 11 de Septiembre de 2008. 


\section{Introducción}

En los pacientes portadores de obesidad mórbida $(\mathrm{OM})$ sometidos a bypass gástrico puede encontrarse patología tumoral benigna o neoplásica, como tumores estromales del tracto gastrointestinal (GIST), leiomiomas, cáncer gástrico y excepcionalmente quistes broncogénicos.

La coexistencia de GIST y obesidad mórbida, ha sido reportada en menos de un $1 \%$ de los pacientes sometidos a cirugía bariátrica ${ }^{1-3}$.

Los quistes broncogénicos del esófago, corresponden a lesiones de origen embriológico, por alteraciones en el desarrollo del intestino anterior, entre las semanas 3 a 7 del crecimiento intrauterino ${ }^{4-7}$. La mayoría de los casos corresponden a pacientes pediátricos, siendo excepcional su hallazgo en adultos.

En pacientes intervenidos con técnica de bypass gástrico, la asociación de leiomioma gástrico con tumores cardiales de origen bronquial no ha sido reportada en la literatura. El propósito del presente estudio es presentar un caso clínico que presenta estas patologías, analizar los métodos diagnósticos disponibles, las alternativas terapéuticas y revisar la literatura.

\section{Caso clínico}

Se presenta una paciente de género femenino de 57 años de edad, portadora de Obesidad Mórbida, candidata a cirugía bariátrica. Presenta peso de 130 $\mathrm{kg}$ y talla $1,58 \mathrm{~m}$, con IMC de 52. Como comorbilidades: Diabetes Mellitus Tipo 1, insulino dependiente, hipertensión severa en tratamiento con 3 drogas, dislipidemia en tratamiento con atorvastatina e hipotiroidismo en tratamiento. Con antecedente de Ecotomografia abdominal, practicada 2 años previos a la cirugía, informada como lesión quística de lóbulo izquierdo del hígado, de $5,5 \mathrm{~cm}$ de diámetro (Figura 1).

$\mathrm{Su}$ estudio preoperatorio incluye TAC de abdomen que muestra lesión subcardial de $70 \times 60 \mathrm{~mm}$ (Figura 2), interpretada como GIST y adenoma suprarrenal de $3 \mathrm{~cm}$, con ausencia de lesiones en hígado. Endoscopía de Esófago, estómago y duodeno (EED) inicial, revela lesión subcardial, de 60 $\mathrm{mm}$, ulcerada (Figura 3). Es tratada por 2 meses con omeprazol y una nueva endoscopía EED no muestra lesiones (Figura 4), con biopsias negativas. Rx EED informa lesión submucosa, subcardial compatible con GIST. El estudio de adenoma suprarrenal, con Resonancia Magnética y determinaciones de catecolaminas, concluye en una lesión no funcionante.
La hipótesis diagnóstica preoperatoria fue: obesidad mórbida (IMC 52), GIST subcardial, adenoma suprarrenal no funcionante, diabetes mellitus insulino dependiente, hipertensión arterial, dislipidemia e hipotiroidismo en tratamiento. Completó estudio preoperatorio, según pauta, incluyendo evaluación siquiátrica, respiratoria, cardiovascular y nutricional y anestésica, obteniendo el pase operatorio de cada especialidad.

Es intervenida practicándose gastrectomía total, por laparotomía, con anastomosis esófago-yeyunal en Y de Roux, y asa de $180 \mathrm{~cm}$, englobando en la pieza las lesiones subcardiales. La lesión suprarrenal no fue resecada, decidiéndose su observación por corresponder a lesión no funcionante. Su evolución postoperatoria fue sin incidentes. Inicia su realimentación oral al día 8 , después de control radiológico de transito esófago-yeyunal que informa ausencia de fístula de la anastomosis esófagoyeyunal y buen paso a distal. Tolera bien aporte oral, se inicia nuevo esquema de manejo de su diabetes, con régimen de gastrectomizado e insulina cristalina y lenta. Es dada de alta al día 13 postoperatorio con tratamiento oral exclusivo y buen control metabólico de su diabetes.

La biopsia intraoperatoria informó la existencia de dos lesiones diferentes, la primera de carácter quístico de $40 \mathrm{~mm}$ de probable origen bronquial y la segunda, sólida, de $1 \mathrm{~cm}$, informada como GIST esofágico, ambas dependientes del plano muscular de la región esófago-gástrica. El informe definitivo de anatomía patológica informa lesión quística abscedada cardial de $40 \mathrm{~mm}$ compatible con quiste broncogénico (Figura 5). El estudio histológico muestra la superficie interna del quiste con un epitelio de tipo respiratorio, cilíndrico ciliado, con denso infiltrado linfo-plasmocitario del estroma. Está rodeado de tejido muscular, derivado de la capa muscular propia del esófago (Figura 6). La segunda lesión corresponde a un leiomioma subcardial de 1 $\mathrm{cm}$, negativa para CD117, CD 34 y positiva para desmina (Figura 7).

Los controles postoperatorios a 4 meses, revelan paciente en buenas condiciones generales, metabólicamente estable, con un episodio intercurrente de infección urinaria y baja de peso satisfactoria, de $30 \mathrm{~kg}$.

\section{Discusión}

El hallazgo de patología tumoral intramural gástrica (TIMG), benigna o maligna, si bien es de baja frecuencia, es un hecho bien establecido en la cirugía bariátrica. Se estima su incidencia en menos de un $1 \%$. 


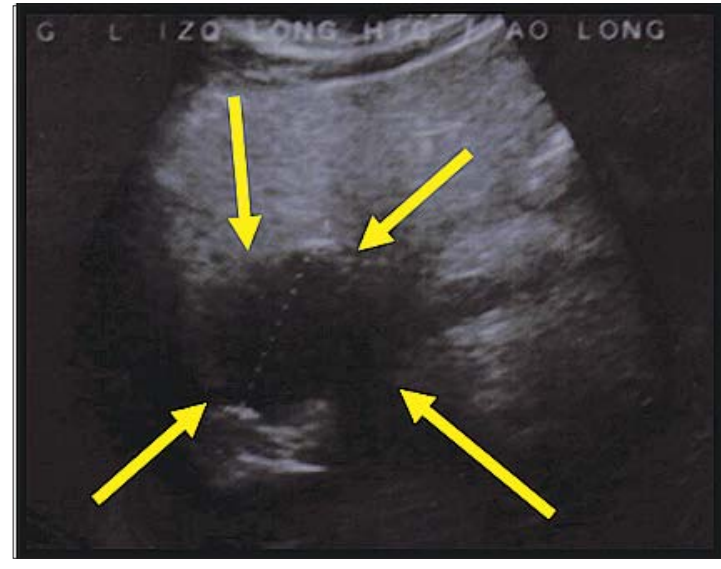

Figura 1. Ecotomografía preoperatoria. Las flechas señalan imagen quística de $55 \mathrm{~mm}$, en relación a lóbulo izquierdo del hígado.

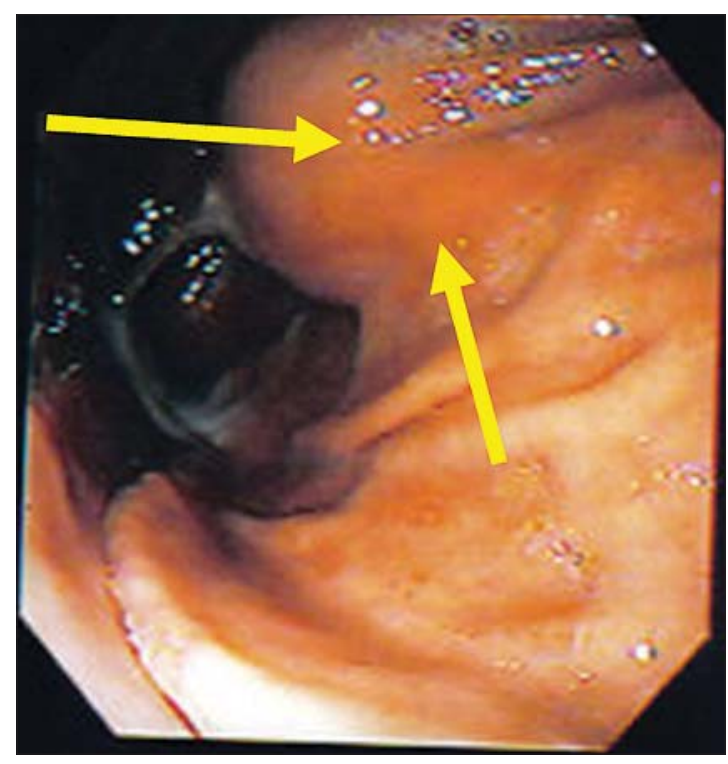

Figura 3. Panendoscopía inicial. Se observa extensa lesión solevantada submucosa de $6 \mathrm{~cm}$, detrás de la cual se encontró lesión ulcerada subcardial de $20 \mathrm{~mm}$.

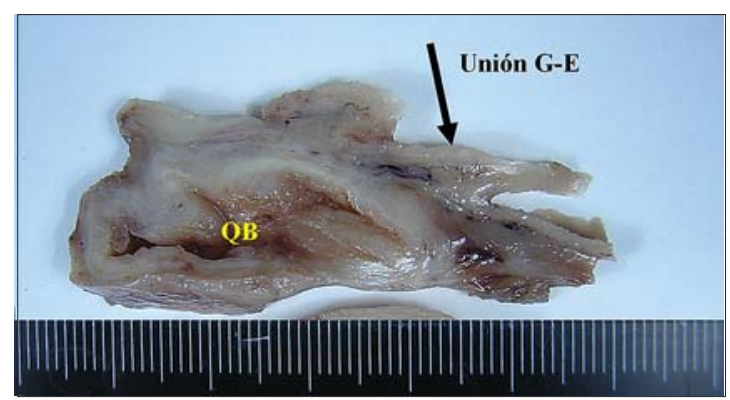

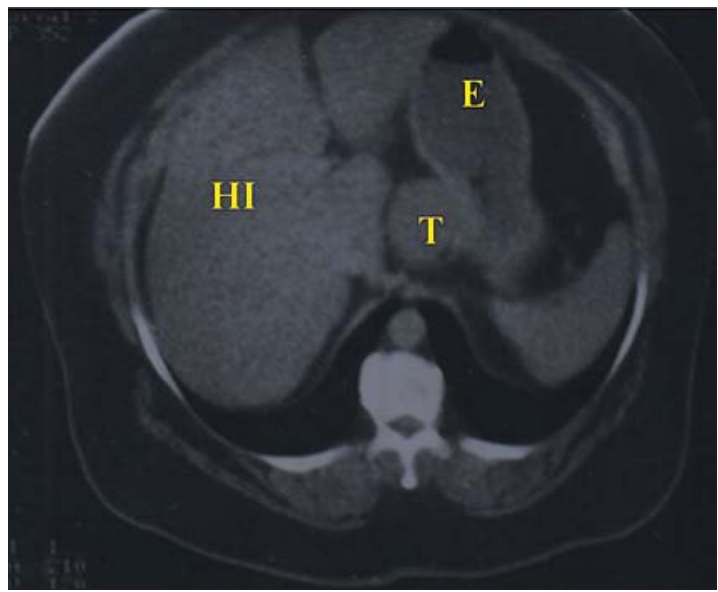

Figura 2. Tomografía Axial Computarizada Preoperatoria. Se observa lesión sólida, de 70x60 mm (T), dependiente de la pared gástrica subcardial (E), claramente separada del hígado (HI).

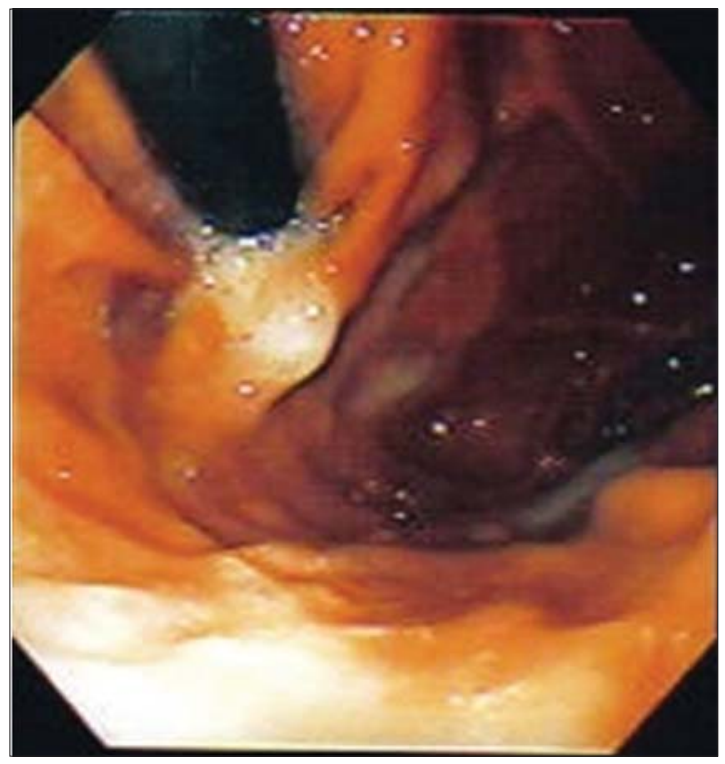

Figura 4. Panendoscopía, control. Se observa región subcardial sin lesiones.

Figura 5. Quiste Broncogénico, aspecto macroscópico. Se observa lesión quística, de 3,5 cm en el espesor de la capa muscular gástrica, bien delimitada. La superficie interna se ve de color rojizo, levemente irregular. En el contorno superior de ambos fragmentos, la flecha señala la unión gastro-esofágica. 


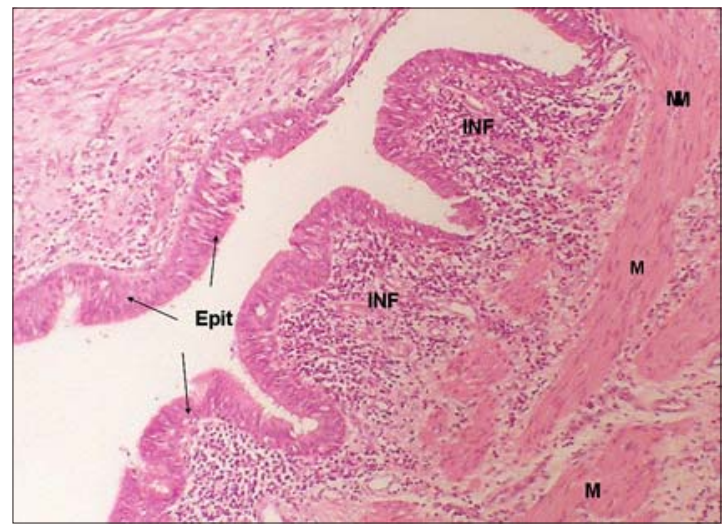

Figura 6. Quiste broncogénico, microscopía. Tinción Hematoxilina-Eosina. Aumento mediano. Superficie interna del quiste con epitelio de tipo respiratorio (Epit), cilíndrico ciliado, con denso infiltrado (INF), linfoplasmocitario del estroma. Está rodeado de tejido muscular (M), derivado de la capa muscular propia del esófago.

Palmer $^{8}$ en una revisión clásica, de 1.660 casos publicados, de tumores intramurales gástricos, los clasifica en: Leiomoma (37 a 56\%), fibroma (17 a $30 \%$ ), neurogénicos, (schwannomas o neurinemomas y neurofibromas) (5 a 16\%), páncreas aberrante $(5$ a $13 \%)$, lipomas (2 a $6 \%)$, tumores de origen vascular (1 a $6 \%$ ) y tumores quísticos (1 a $5 \%$ ).

En la experiencia nacional la mayor frecuencia también la representan los leiomiomas con alrededor de la mitad de los casos9.

En la cirugía de la patología gástrica benigna se han descrito con una incidencia de $0,2 \%$. Los criterios clásicos los clasifican en: leiomiomas (42\%), neurilemoma $(22,5 \%)$, páncreas aberrante $(12,9 \%)$, fibroma $(6,5 \%)$, lipoma $(6,5 \%)$ y hemangiopericitoma $(3,2 \%)^{9}$.

Hasta hace un par de décadas, se consideraba que la mayoría de los tumores mesenquimáticos del estomago tenían origen en el músculo liso o perineural. En 1983, Mazur y Clark ${ }^{10}$, acuñaron el término GIST (gastrointestinal stromal tumours). Se ha sugerido su origen de las células intersticiales de Cajal ${ }^{11}$.

A pesar de su baja frecuencia, los GIST son los tumores mesenquimáticos mas frecuentes del tracto gastrointestinal. La importancia clínica, radica en el potencial maligno que se presenta en un tercio de los $\operatorname{casos}^{12}$.

La resistencia de estos tumores a las clásicas terapias de quimioterapia y radioterapia, se constituyó en un desafío terapéutico. En la actualidad la identificación de factores pronósticos y el desarro-

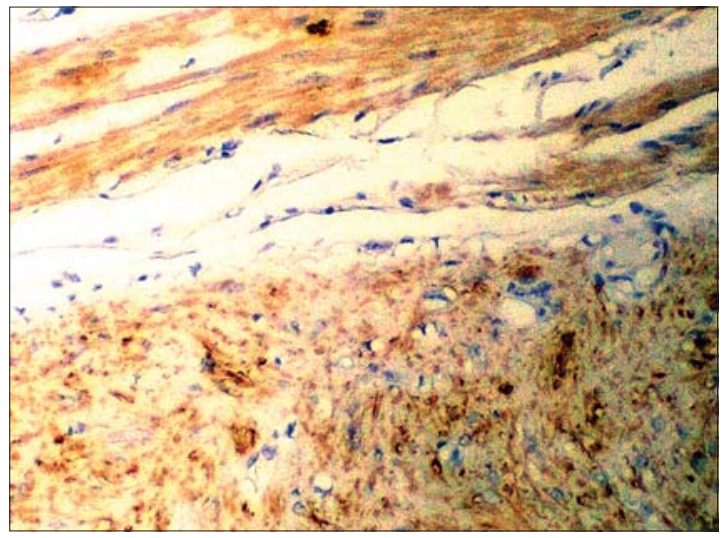

Figura 7. Leiomioma gástrico. Inmunohistoquímica para desmina. Inmunohistoquímica para marcador desmina (Muscular), positivo en las células neoplásicas y en capa muscular gástrica, las que se observan de color café.

1lo de terapias anticancerosas, basadas en estrategias moleculares blanco (target) con fármacos como el Imatinib, ofrecen una nueva perspectiva y abren un interesante campo en la terapia antineoplásica. El Imatinib ${ }^{R}$ es un derivado 2-fenilaminopirimidina que actúa como un inhibidor especifico de la proteína tirosin-kinasa, que inhibe la proliferación e induce la apoptosis de las células tumorales de los tumores estromales gastrointestinales (GIST), que expresan una mutación activante de los receptores c-kit ${ }^{12-14}$.

La presencia de TIMG, son un hecho bien documentado $23,8,9$, si bien su incidencia global no supera el $1 \%$ de los pacientes sometidos a cirugía bariátrica y, siendo la mayoría de los casos hallazgos intraoperatorios, se recomienda efectuar una cuidadosa revisión gástrica durante la cirugía ${ }^{3}$, antes de confeccionar el reservorio gástrico. Zeni ${ }^{2}$, enfatiza el rol de la endoscopía de EED, como parte del estudio preoperatorio en estos pacientes. La mayor parte de estas lesiones se encuentran en tercio proximal, cara anterior del estómago ${ }^{3}$.

Liberatt ${ }^{1}$, en un meta-análisis de 14 estudios que incluyen 728 pacientes portadores de GIST postula que, ante la imposibilidad de determinar su futuro comportamiento biológico debe recomendarse la resección de las lesiones, con márgenes mayores a $1 \mathrm{~cm}$.

Dentro de los TIMG, las lesiones más frecuentes son los leiomiomas ${ }^{8,9}$, en casi la mitad de los casos. Los criterios actuales de diagnóstico histológico, utilizando reacciones histoquímicas, han reclasificado a un porcentaje de estas lesiones como 
GIST $^{15-21}$. El presente caso presenta reacción CD117 (receptor de factor de crecimiento tirosinkinasa) y CD-34, ambas negativas, lo que se interpreta como lesión no GIST; sin embargo, un $5 \%$ de los GIST pueden ser negativos con estas técnicas histoquímicas, y presentar mutaciones oncogénicas a nivel de los receptores de factores de crecimientos derivados de plaquetas (PDGFRA) ${ }^{21}$, lo que podría corresponder al presente caso.

La paciente que se presenta corresponde a una Obesa Mórbida severa (IMC 52), con múltiples comorbilidades. Sus evaluaciones previas, con ecotomografía abdominal, informaban un quiste hepático de lóbulo izquierdo, lo que probablemente correspondía a las lesiones resecadas. El diagnóstico preoperatorio de una lesión subcardial compatible con un GIST, en la TAC, con ausencia de lesiones hepáticas, concuerda con lo planteado por Liberatti ${ }^{1}$, en el sentido de que el examen de imágenes de mayor rendimiento para estas lesiones es la TAC.

Thomas $^{22}$, recomienda la Tomografía por emisión de positrones, y De Simone ${ }^{7}$, plantea la utilidad de la endosonografía en el diagnóstico de lesiones sólidas y quísticas.

La interpretación como GIST, de la lesión subcardial encontrada en la tomografía en el presente caso, puede corresponder a la presencia de un fluido altamente viscoso, con una densa estructura proteica, en el interior del quiste broncogénico, como reporta $\mathrm{Melo}^{4}$, en el caso de una paciente asintomática en relación al tubo digestivo, en la que se efectuó estudio con TAC de tórax por la presencia de un lipoma de mama, encontrando una lesión subcardial de $6 \mathrm{~cm}$ interpretada inicialmente como GIST, y que en la anatomía patológica resultó ser un quiste broncogénico, tal como en el presente caso.

Los quistes broncogénicos son de excepcional incidencia en adultos, y la mayoría de los casos reportados corresponden a pacientes pediátricos. Nobuhara ${ }^{5}$ en una revisión de 68 pacientes pediátricos, reporta la aparición de complicaciones de estos quistes, como ulceraciones gástricas como la presentada por la paciente actual, hemorragias digestivas, que pueden llegar a ser exanguinizantes, infección de la cavidad quística, y obstrucción de la vía aérea o digestiva, por compresión extrínseca. Recomienda la extirpación de estos quistes aún en los pacientes asintomáticos, por el riesgo de presentar algunas de estas complicaciones, que en su serie originaron una mortalidad del $10 \%$.

La etiología de los quistes broncogénicos, es de origen embriológico, secundaria a alteraciones en el desarrollo del intestino anterior, entre la tercera y séptima semana de gestación ${ }^{7}$. Esta etiología es también común a los quistes de duplicación esofágica ${ }^{20}$.
Este mecanismo explica porque la mayoría de los casos publicados, corresponden a pacientes pediátricos, y lo excepcional de su presentación en adul$\operatorname{tos}^{5,23-26}$

El diagnóstico preoperatorio de una lesión subcardial que presentó una extensa ulceración comprobada endoscópicamente, nos llevó a la decisión de plantear una gastrectomía total, resecando en block la lesión para evitar dejar un reservorio gástrico con una curvatura menor potencialmente dañada y expuesta a nuevas ulceraciones, o con daño en su irrigación al haber efectuado una resección de la lesión subcardial por separado. La gastrectomía total, en pacientes portadores de GIST gástrico, también ha sido planteada como una alternativa, en casos seleccionados, por De $\mathrm{Mateo}^{27} \mathrm{y}$ Fujimoto ${ }^{18}$.

El caso presentado corresponde a una paciente portadora de obesidad mórbida severa, portadora de múltiples comorbilidades, en la cual se asocian patologías de baja frecuencia como el leiomioma subcardial y los adenomas suprarrenales a un cuadro excepcionalmente poco frecuente, como son los quistes broncogénicos. La asociación de estas patologías no ha sido reportada previamente hasta donde hemos podido revisar la literatura. El diagnóstico preoperatorio de ambas lesiones permitió diseñar una estrategia quirúrgica adecuada al caso.

\section{Referencias}

1. Palmer ED. Benign intramural tumors of the stomach: a review with special reference in gross pathology. Medicine 1951; 30: 81-88.

2. Csendes A, Braghetto I. Patología gástrica benigna. Editorial Andres Bello, Santiago 1985, 291-299.

3. Mazur MT, Clark HB. Gastric stromal tumors. Reappraisal of histogenesis. Am J Surg Pathol 1983; 7: 507-519.

4. Kindblom LG, Remotti HE, Aldenborg F, MeisKindblom JM. Gastrointestinal pacemaker cell tumour (GIPACT): gastrointestinal stromal tumours show phenotypic characteristics of the interstitial cells of Cajal. Am J Pathol 1998; 152: 1259-1269.

5. Demetri GD, von Mehren M, Blanke CD, Van den Abbeele AD, Eisenberg B, Roberts PJ, et al. Efficacy and safety of Imatinib mesylate in advanced gastrointestinal tumours. N Engl J Med 2002; 347: 472-480.

6. Demetri, G. Targeting the molecular pathophysiology of gastrointestinal stromal tumours with imatinib. Mechanisms, successes and challenges to rational drug development. Hematol Oncol Clin North Am 2002; 16: $1115-1124$

7. Joensuu H, Fletcher C, Dimitrijevic S, Silberman S, 
Roberts P, Demetri G. Management of malignant gastrointestinal stromal tumours. Lancet Oncol 2002; 3: 655-664.

8. Zeni TM, Frantzides CT, Mahr C, Denham EW, Meiselman M, Goldberg MJ, et al. Value of preoperative upper endoscopy in patients undergoing laparoscopic gastric bypass. Obes Surg 2006; 16: 142146.

9. Sánchez BR, Morton JM, Curet MC, Alami RZ, Safadi BY. Incidental Finding of Gastrointestinal Stromal Tumors (GIST) during Laparoscopic Gastric Bypass. Obesity Surgery 2005; 15: 1384-1388.

10. Liberati G, Lucchetta MC, Petraccia L, Nocchi S, Rosentzwig R, De Matteis A, et al. Meta-analytical study of gastrointestinal stromal tumors (GIST) Clin Ther 2003; 154: 85-91.

11. Melo N, Pitman MB, Rattner DW. Bronchogenic cyst of the gastric fundus presenting as a gastrointestinal stromal tumor. J Laparoendosc Adv Surg Tech A 2005; 15: 163-165.

12. Nobuhara KK, Gorski YC, La Quaglia MP, Shamberger RC. Bronchogenic cysts and esophageal duplications: common origins and treatment. J Pediatr Surg 1997; 32: 1408-1413.

13. Martin ND, Kim JC, Verma SK, Rubin R, Mitchell DG, Bergin D, et al. Intra-abdominal Esophageal Duplication Cysts: A Review. J Gastrointest Surg 2007; 11: 773-777.

14. De Simone M, Cioffi U. Leiomyomas and extramucosal cysts of the esophagus in adults. The clinical picture and surgical therapy. Minerva Chir 1999; 54: 15-25.

15. Nilsson B, Bümming P, Meis-Kindblom JM, Odén A, Dortok A, Gustavsson B, et al. Gastrointestinal stromal tumors: The incidence, prevalence, clinical course, and prognostic in the preimatinib mesylate era. Cancer 2005; 103: 821-829.

16. Braghetto I, Parada F, Cardemil G, Csendes A, Fernández E, Korn $\mathrm{O}$ y cols. Tumores gastrointestinales (GIST): Experiencia del Departamento de Cirugía del Hospital Clínico de la Universidad de Chile entre 1999 y 2005. Rev Méd Chile 2007; 135: 551-557.
17. Roggen JF, Velthuysen MF, Hogendoorn PW. The histopathological differential diagnosis of gastrointestinal stromal tumors. J Clin Pathol 2001; 54: 96-103.

18. Fujimoto Y, Nakanishi Y, Yoshimura K. Clinicopathologic study of primary malignant gastrointestinal stromal tumor of the stomach, with special reference to prognostic factors: analysis of results in 140 surgically resected patients. Gastric Cancer 2003; 6: 39-48.

19. Miettinen M, Majidi M, Lasota J. Pathology and diagnostic criteria of gastrointestinal stromal tumors (GIST): a review. Eur J Cancer 2002; 38: S39-51.

20. Pidhorecky I, Cheney RT, Kraybill WG. Gastrointestinal stromal tumors: current diagnosis, biologic behavior, and management. Ann Surg Oncol 2000; 7: 705-712.

21. Bucher P, Villiger P, Egger JF, Buhler LH, Morel P. Management of gastrointestinal stromal tumors: from diagnosis to treatment. Swiss Med Wkly 2004; 134: 145-153.

22. Thomas A, Singh A. Role of Positron Emission Tomographic Imaging in Gastrointestinal Stromal Tumors. Appl Radiol 2005; 34: 10-17.

23. Cioffi U, Bonavina L, De Simone M, Santambrogio L, Pavoni G, Testori A, et al. Presentation and surgical management of bronchogenic and esophageal duplication cysts in adults. Chest 1998; 113: $1492-$ 1496.

24. Zaunbauer W, Amsler UJ, Haertel M. Bronchogenic cyst. A rare benign esophageal tumor. Radiology 1996; 36: 991-995.

25. Ribet M, Gosselin B, Watine O, Pruvot FR, Sault MC, Jarry JM. Congenital cysts of the esophageal wall with a respiratory type mucosa. Ann Chir 1989; 43: 692698.

26. Hocking M, Young DG. Duplication of the alimentary tract. Br J Surg 1981; 68: 92-98.

27. DeMatteo RP, Lewis JJ, Leung D. Two hundred gastrointestinal stromal tumors: recurrence patterns and prognostic factors for survival. Ann Surg 2000; 231: $51-58$. 